\title{
The applications of machine learning in plastic and reconstructive surgery: protocol of a systematic review
}

\author{
Angelos Mantelakis ${ }^{1,2^{*}+}$ and Ankur Khajuria ${ }^{3,4+}$
}

\begin{abstract}
Background: Machine learning, a subset of artificial intelligence, is a set of models and methods that can automatically detect patterns in vast amounts of data, extract information and use it to perform various kinds of decision-making under uncertain conditions. This can assist surgeons in clinical decision-making by identifying patient cohorts that will benefit from surgery prior to treatment. The aim of this review is to evaluate the applications of machine learning in plastic and reconstructive surgery.
\end{abstract}

Methods: A literature review will be undertaken of EMBASE, MEDLINE and CENTRAL (1990 up to September 2019) to identify studies relevant for the review. Studies in which machine learning has been employed in the clinical setting of plastic surgery will be included. Primary outcomes will be the evaluation of the accuracy of machine learning models in predicting a clinical diagnosis and post-surgical outcomes. Secondary outcomes will include a cost analysis of those models. This protocol has been prepared using the Preferred Items for Systematic Review and Meta-Analysis Protocols (PRISMA-P) guidelines.

Discussion: This will be the first systematic review in available literature that summarises the published work on the applications of machine learning in plastic surgery. Our findings will provide the basis of future research in developing artificial intelligence interventions in the specialty.

Systematic review registration: PROSPERO CRD42019140924

Keywords: Artificial intelligence, Machine learning, Deep learning, Plastic surgery, Big data

\section{Background}

In the era of big data, the plethora of efforts towards gathering and analysing patient data in large scale is rapidly increasing [1]. Amongst others, these efforts try to improve the diagnosis of diseases and the prediction of post-treatment outcomes using large amounts of data from past cases. The analysis of this vast amount of information, however, is beyond the capabilities of

\footnotetext{
* Correspondence: aggelosmantelakis@yahoo.com

${ }^{+}$Angelos Mantelakis and Ankur Khajuria contributed equally to this work.

'Lewisham and Greenwich NHS Trust, London, UK

¿London, UK

Full list of author information is available at the end of the article
}

traditional statistical methods previously used in academic medicine [2].

Machine learning, a subset of artificial intelligence, is the set of models and methods that can automatically detect patterns in vast amounts of data, extract information and use it to perform various kinds of decisionmaking under uncertain conditions [3]. These models have the potential of two principally distinct functions: supervised and unsupervised learning (termed "deep learning"). Supervised learning involves the creation and optimisation of statistical models which aim to predict an outcome using information from past cases [2, 4]. In contrast, unsupervised learning aims to identify patterns

(c) The Author(s). 2020 Open Access This article is licensed under a Creative Commons Attribution 4.0 International License, which permits use, sharing, adaptation, distribution and reproduction in any medium or format, as long as you give appropriate credit to the original author(s) and the source, provide a link to the Creative Commons licence, and indicate if changes were made. The images or other third party material in this article are included in the article's Creative Commons licence, unless indicated otherwise in a credit line to the material. If material is not included in the article's Creative Commons licence and your intended use is not permitted by statutory regulation or exceeds the permitted use, you will need to obtain permission directly from the copyright holder. To view a copy of this licence, visit http://creativecommons.org/licenses/by/4.0/ The Creative Commons Public Domain Dedication waiver (http://creativecommons.org/publicdomain/zero/1.0/) applies to the data made available in this article, unless otherwise stated in a credit line to the data. 
in previously seemingly random data and generate novel associations [2, 4, 5].

Healthcare professionals have been quick to adopt these emerging technologies to improve patient outcomes [5]. Examples include machine learning models created to identify clinical diagnoses, which perform to the level of expert clinicians in identifying acute cerebral ischaemia, malignant skin lesions and lung cancer subtypes [6-8]. In the field of surgery, this technology has demonstrated a unique potential in predictive postoperative success and complication rate in procedures such as traumatic brain injury, cervical spine fusion and glioma removal, amongst others [9-11].

This technology has the potential to provide clinically relevant information across many areas of plastic surgery. In burn surgery, machine learning has been used to predict whether complete wound healing will require more or less than 14 days with an accuracy rate of $86 \%$ [12]. In the field of microsurgery, authors have been able to predict surgical site infections following free flap reconstruction in head and neck cancer with a sensitivity of $81 \%$ and specificity of $61 \%$ through using artificial intelligence neural networks [13]. Further, machine learning has also been applied in aesthetic surgery research, where using supervised learning, the authors were able to extract potential beauty-determining facial features to guide pre-operative planning [14].

The aim of this review is to systematically analyse the available literature on the applications of deep learning in plastic surgery. Data collected will be used to provide an up-to-date overview of the potential utility of this technology in the specialty and suggest future directions of further research.

\section{Methods}

\section{Aim}

This systematic review is intended to evaluate the clinical applications of machine learning models in the field of plastic and reconstructive surgery and to determine areas of future research on this technology.

\section{Protocol and registration}

This protocol is registered in the Prospective Register of Ongoing Systematic Reviews (PROSPERO) CRD42019140924 and adheres to the Preferred Reporting Items for Systematic Review guidelines and Meta-Analysis Protocols (PRISMA-P 2015) [15] [Additional File].

\section{Search strategies}

All studies published between 1990 and the date of the search will be considered for review.

We will perform a comprehensive search of MEDLINE (OVID SP), EMBASE (OVID SP), Science Citation Index, ClinicalTrials.gov and CENTRAL. A combination of free text and Medical Subject Headings (MeSH) terms will be used. An example search strategy in MEDLINE is the following:

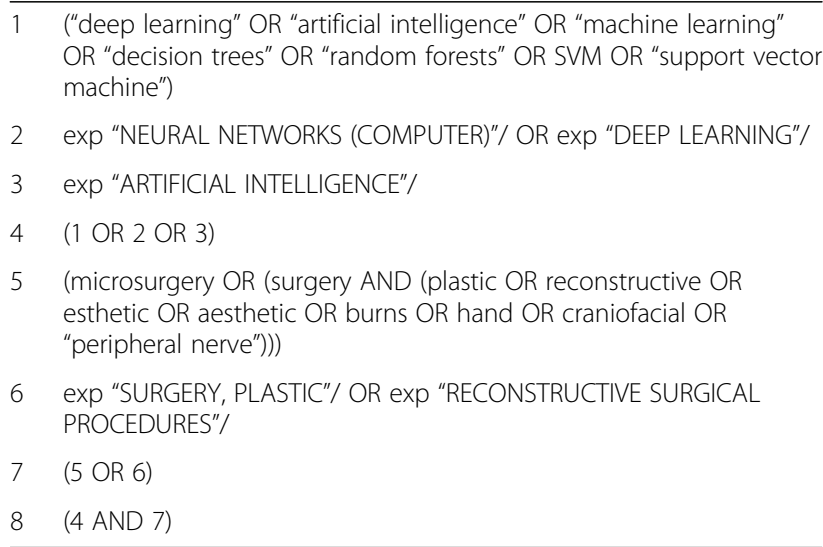

\section{Identification and selection of studies}

Following database searching, studies will be populated into Endnote X7 library (Clarivate Analytics, USA). There will be two stages of screening, carried by two independent reviewers using pre-specified criteria. The search results, including abstracts, full-text articles and record of reviewer's decisions, including reasons for exclusion, will be recorded.

1. Stage 1: Title and abstract review. This will be carried out by the two independent researchers by adhering to the set eligibility criteria. Any discrepancies will be resolved through a consult by a third reviewer.

2. Stage 2: Studies included will undergo full-text review by the same independent reviewers. Any discrepancies will be resolved through a consult to a third reviewer.

\section{Eligibility criteria \\ Types of studies}

Any primary studies (including case reports), which assess the prediction rate of deep learning models in diagnosis of disease or post-operative outcomes in plastic surgery, either on its own or compared to other techniques, will be included. There will be no geographical restriction. Our exclusion criteria include studies utilising machine learning without clinical data, non-English language articles and review articles.

\section{Types of study participants}

We will include clinical data from adult participants (>18 years old) with conditions requiring plastic or reconstructive surgery. Data from animal studies will be excluded. 


\section{Types of interventions}

The studies considered will present artificial intelligence models utilising deep learning as an intervention with the aim to provide a diagnosis of a clinical presentation, or a clinical prognosis of a plastic surgery intervention. The intervention may be used by itself or in combination with other methods. Since this technology is new, there is no single best deep learning model, and because of the versatility of conditions treated in plastic surgery, it is expected that various different models will be identified in our review.

\section{Outcomes}

\section{Primary outcomes}

The primary outcomes will be the evaluation of deep learning models on two distinct functions. The first function is the accuracy of providing a clinical diagnosis. Studies must have a defined clinical condition for which the model is designed to identify. The accuracy of performing this task (either on its own or in assistance with a clinician) will be collected.

The second primary outcome will be the accuracy of prediction of post-operative outcomes and complications of plastic surgery interventions. In order to qualify, studies will need to have created a model to predict a particular clinical outcome (for example, probability of post-operative wound infection), with data collected prospectively or retrospectively.

In both settings, the model's accuracy will be assessed by the reported specificity, sensitivity, positive predictive value and negative predictive value of performing the named task.

\section{Secondary outcomes}

The secondary outcomes will include cost analysis of the deep learning models. Further, outcomes of studies that have utilised deep learning models as a treatment for a clinical condition (for example, neuroprosthesis) will also be collected.

\section{Data extraction, collection and management}

After the study selection is completed, the two reviewers will independently extract data using a standardised data extraction form. Any disagreements and differences will be resolved by discussion with a third reviewer.

The following data will be extracted:

1. Study characteristics (authors, year of publication, study design)

2. Patient demographics (number of participants, sex, mean age)

3. Indication of application of the software model (prediction of a diagnosis or treatment outcome)

4. Software characteristics
5. Outcomes (specificity, sensitivity, positive predictive value and negative predictive value of forming a

diagnosis; predicting rates of overall survival, treatment success, post-operative function, aesthetic outcome, complications and recurrence)

6. Complications or adverse events reported

\section{Risk of bias}

The risk of bias in the selected randomised controlled trials will be evaluated by the two independent reviewers through utilising the Cochrane Collaboration Risk of Bias tool [16]. The methodological quality will be assessed based on appropriate participant selection and randomisation, blinding of participants and reviewers, attrition, selective reporting and others. An overall grading of low, medium or high risk of bias will then be allocated. For non-randomised trials, the ROBINS-I (Risk of Bias in Non-randomised Studies-of Interventions) will be utilised [17]. For quantitative studies in which the ROBINS-I is not applicable, risk of bias assessment will be undertaken using the Quality Assessment Tool for Quantitative studies [18]. Case reports will be included as part of screening for all available evidence; however, they are inherently at high risk of bias and this will be considered during the assessment of the quality of overall evidence.

The risk of bias in the performance of deep learning models will be evaluated using the QUADAS-2 (Quality Assessment for Diagnostic Accuracy Studies) tool [19]. This will examine the process of patient selection and the conduction and interpretation of the index test and reference standard. An overall risk of bias will be subsequently allocated (high, low, or unclear).

\section{Data analysis}

The two independent reviewers will explore the heterogeneity between the studies using the Review Manager 5.3 provided by the Cochrane Collaboration (1). Potential sources of heterogeneity include the deep learning software, its intention (diagnosis or treatment), the treatment indication and population. A narrative review will be carried out structured around the intervention and outcome of interest. A quantitative analysis (meta-analysis) will be performed if sufficient homogeneous studies in terms of design and outcomes measures are identified.

Statistical heterogeneity will be assessed using the $I^{2}$ statistic [20]. A random-effects model will be employed for heterogenous cohorts $\left(I^{2}>50 \%\right)$. The quality of overall evidence will be assessed using The Grading of Recommendations Assessment, Development and Evaluation (GRADE) approach [21]. Sensitivity analysis will be attempted based on the study quality. This may be repeated after removal of poor-quality studies that may affect the overall effect estimate. 


\section{Discussion}

Due to the incredible potential of machine learning to process vast amounts of patient information and provide clinically relevant predictions, it is important for plastic surgeons to be informed with the up-to-date applications of this technology in the specialty. The aim of our review is to systematically evaluate the current evidence of this technology in the clinical setting and to discuss the future prospects of machine learning in guiding patient management. To the authors' knowledge, this is the first systematic review to evaluate the applications of artificial intelligence in plastic surgery.

\section{Supplementary information}

Supplementary information accompanies this paper at https://doi.org/10. 1186/s13643-020-01304-X.

\section{Additional file 1}

\section{Abbreviations}

CENTRAL: Cochrane Central Register of Controlled Trials; EMBASE: Excerpta Medica Database; GRADE: Grading of Recommendations Assessment, Development and Evaluation; PRISMA-P: Preferred Reporting Items for Systematic Review and Meta-Analysis Protocols; QUADAS-2: Quality Assessment for Diagnostic Accuracy Studies; ROBINS-I: Risk of Bias in Nonrandomised Studies-of Interventions

\section{Acknowledgements}

We would like to thank Dr Yannis Assael for his invaluable technical support and guidance in helping us understanding the function and capabilities of deep learning in machine learning models.

\section{Authors' contributions}

Both authors contributed equally to the conception of the protocol and study design, reviewed this report and approved the final manuscript.

\section{Funding}

No funding was received for this study.

\section{Availability of data and materials}

The datasets generated and/or analysed during the current study are available in the MEDLINE (OVID SP), EMBASE (OVID SP), Science Citation Index, ClinicalTrials.gov and CENTRAL repositories. https://ovidsp.ovid.com/

http://mjl.clarivate.com/cgi-bin/jrnlst/jloptions.cgi?PC=K https://clinicaltrials.gov/

https://www.cochranelibrary.com/central/about-central

\section{Ethics approval and consent to participate}

Not applicable

\section{Consent for publication}

Not applicable.

\section{Competing interests}

The authors declare that they have no competing interests.

\section{Author details}

${ }^{1}$ Lewisham and Greenwich NHS Trust, London, UK. ${ }^{2}$ London, UK. ${ }^{3}$ Kellogg College, University of Oxford, Oxford, UK. ${ }^{4}$ Department of Surgery and Cancer, Imperial College London, London, UK.
Received: 18 September 2019 Accepted: 20 February 2020

Published online: 28 February 2020

\section{References}

1. Lee $\mathrm{CH}$, Yoon HJ. Medical big data: promise and challenges. Kidney Res Clin Pract. 2017;36(1):3.

2. Kanevsky J, Corban J, Gaster R, Kanevsky A, Lin S, Gilardino M. Big data and machine learning in plastic surgery: a new frontier in surgical innovation. Plastic Reconstr Surg. 2016;137(5):890e-7e.

3. Murphy KP. Machine learning: a probabilistic perspective. Cambridge: MIT press; 2012.

4. Celtikci E. A systematic review on machine learning in neurosurgery: the future of decision-making in patient care. Turk Neurosurg. 2018 Jan 1;28(2): $167-73$

5. Noorbakhsh-Sabet N, Zand R, Zhang Y, Abedi V. Artificial intelligence transforms the future of healthcare. Am J Med. 2019;31.

6. Abedi V, Goyal N, Tsivgoulis G, et al. Novel screening tool for stroke using artificial neural network. J Stroke. 2017:48:1678-81.

7. Cruz-Roa AA, Arevalo Ovalle JE, Madabhushi A, González Osorio FA. A deep learning architecture for image representation, visual interpretability and automated basal-cell carcinoma cancer detection. Med Image Comput Comput Interv. 2013:16:403-10.

8. Lehman CD, Yala A, Schuster T, et al. Mammographic breast density assessment using deep learning: clinical implementation. Radiology. 2019; 290:52-8.

9. Shi HY, Hwang SL, Lee KT, Lin CL. In-hospital mortality after traumatic brain injury surgery: a nationwide population-based comparison of mortality predictors used in artificial neural network and logistic regression models. J Neurosurg. 2013;118:746-52.

10. Arvind V, Kim JS, Oermann EK, Kaji D, Cho SK. Predicting surgical complications in adult patients undergoing anterior cervical discectomy and fusion using machine learning. Neurospine. 2018;15(4):329.

11. Macyszyn L, Akbari H, Pisapia JM, Da X, Attiah M, Pigrish V, Bi Y, Pal S, Davuluri RV, Roccograndi L, Dahmane N, Martinez-Lage M, Biros G, Wolf RL, Bilello M, O'Rourke DM, Davatzikos C. Imaging patterns predict patient survival and molecular subtype in glioblastoma via machine learning techniques. Neuro Oncol. 2016:18:417-25.

12. Yeong EK, Hsiao TC, Chiang HK, Lin CW. Prediction of burn healing time using artificial neural networks and reflectance spectrometer. Burns. 2005;31: 415-20.

13. Kuo PJ, Wu SC, Chien PC, Chang SS, Rau CS, Tai HL, Peng SH, Lin YC, Chen YC, Hsieh HY, Hsieh CH. Artificial neural network approach to predict surgical site infection after free-flap reconstruction in patients receiving surgery for head and neck cancer. Oncotarget. 2018:9(17):13768.

14. Gunes H, Piccardi M. Assessing facial beauty through proportion analysis by image processing and supervised learning. Int J Human Comput Stud. 2006; 64(12):1184-99.

15. Moher D, Shamseer $L$, Clarke $M$, et al. Preferred reporting items for systematic review and meta-analysis protocols (PRISMA-P) 2015 statement. Syst Rev. 2015:4:1.

16. Higgins Julian P T, Altman Douglas G, Gøtzsche Peter C, Jüni Peter, Moher David, Oxman Andrew D, et al. The Cochrane Collaboration's tool for assessing risk of bias in randomised trials BMJ. 2011;343:d5928.

17. Sterne JA, Hernan MA, Reeves BC, Savovic J, Berkman ND, Viswanathan M, et al. ROBINS-l: a tool for assessing risk of bias in non-randomised studies of interventions. BMJ. 2016;355:14919.

18. Armijo-Olivo S, Stiles CR, Hagen NA, Biondo PD, Cummings GG. Assessment of study quality for systematic reviews: a comparison of the Cochrane Collaboration Risk of Bias Tool and the Effective Public Health Practice Project Quality Assessment Tool: methodological research. J Eval Clin Pract. 2012;18(1):12-8.

19. Whiting P, Rutjes AW, Reitsma JB, Bossuyt PM, Kleijnen J. The development of QUADAS: a tool for the quality assessment of studies of diagnostic accuracy included in systematic reviews. BMC Med Res Methodol. 2003;3(1):25.

20. Higgins JP, Thompson S, Deeks J, Altman DG. Measuring inconsistency in meta-analyses. BMJ. 2003;327:557-60.

21. Atkins D, Best D, Briss PA, Eccles M, Falck-Ytter Y, Flottorp S, et al. Grading quality of evidence and strength of recommendations. BMJ. 2004:328:1490.

\section{Publisher's Note}

Springer Nature remains neutral with regard to jurisdictional claims in published maps and institutional affiliations. 